\title{
Analysis of Imaging and Pathological Features in 12 Cases of Schwannomatosis and Literature Review
}

\section{Cheng-wei Kang ( $\sim$ kangarooqq@163.com )}

West China School of Medicine: Sichuan University West China Hospital https://orcid.org/0000-00034834-8957

\section{Li-xue Wu}

Sichuan University West China College of Public Health: Sichuan University West China School of Public Health

\section{Xiao-bing Pu}

Sichuan University West China College of Public Health: Sichuan University West China School of Public Health

\section{Chang-chao Dong Dong}

Sichuan University West China College of Public Health: Sichuan University West China School of Public Health

\section{Gang Tan}

Sichuan University West China College of Public Health: Sichuan University West China School of Public Health

\section{Zhao-kui Yan}

Sichuan University West China College of Public Health: Sichuan University West China School of Public Health

\section{Jiang Shen}

Sichuan University West China College of Public Health: Sichuan University West China School of Public Health

\section{Lei Liu}

West China School of Medicine: Sichuan University West China Hospital https://orcid.org/0000-00022184-9463

\section{Research Article}

Keywords: Schwannomatosis, Imaging features, Pathology, Immunohistochemistry, Differential diagnosis

Posted Date: May 26th, 2021

DOl: https://doi.org/10.21203/rs.3.rs-539940/v1

License: (a) (i) This work is licensed under a Creative Commons Attribution 4.0 International License. Read Full License 
Page $2 / 18$ 


\section{Abstract}

Objective We analyzed US, CT, and MR images plus the pathological characteristics of 12 patients with pathologically confirmed schwannomatosis to explore the relationship between imaging and pathology.

Method A retrospective analysis was undertaken over a 10-year period (01/01/2000-31/12/2019) of 12 patients undergoing Imaging and pathological examination for schwannomatosis, and the relevant literature was reviewed.

Results The median age at diagnosis was 39.4 years (range, 22-56 years). We included the mean diameter of 36 lesions $(5.10 \pm 0.84 \mathrm{~cm}$; range, $0.5-9.1 \mathrm{~cm})$ in the focal observation. US showed that the mass boundary was clear, and most of the masses were low echo. CT showed that tumors were low density; the plain-scan CT value was 22-35 HU and enhancement amplitude 10-30 HU. MRI showed that most of the tumors were low or medium signal on T1-weighted image (T1WI) and high signal on T2Wl; enhancement could be homogeneous or inhomogeneous. We could see the cellular Antoni A and myxoid Antoni B areas of the microscopic morphology. According to the proportions of the two areas, masses could be divided into four types: I, II, III and IV. Immunohistochemical (IHC) staining showed that the expression of S-100 protein was diffusely positive (36/36).

Conclusions Imaging examination in schwannomatosis is helpful for localization and characterization of focus, as well as observation of the relationship between the tumor and the surrounding tissue structure. However, the specific diagnosis should also be based on pathological manifestations, IHC results and genetic analysis. Characteristics of mass imaging correlate with pathological changes in the mass.

\section{Introduction}

Schwannomatosis is a syndrome that is characterized by multiple peripheral schwannomas and can be sporadic or familial in nature. It is reported that the incidence rate is approximately one in 140,000$150,000[1,2]$. Painless masses are the most common clinical manifestation. In one study, $89 \%$ of schwannomatosis patients had peripheral schwannoma, $74 \%$ had spinal schwannoma and $9 \%$ had intracranial tumor that did not affect the vestibular nerve[3]. The highest priority in the diagnosis of schwannomatosis is to distinguish it from neurofibromatosis type 2 (NF2). About one-third of schwannomatosis patients carry a mutation of leucine zipper transcription regulator 1 (LZTR1), which is located at 22q11.2 and centromeric to smarcb1, a gene that also factors into differential diagnosis between schwannomatosis and NF2, but in many clinical cases the mutant gene cannot be found. Diagnosis of schwannomatosis is therefore usually based on clinical examination, specifically the clinical criteria of $\geq 2$ non-intradermal schwannomas and the absence of bilateral vestibular schwannoma. Imaging and pathology are part of the diagnostic criteria to some degree for this syndrome. The use of imaging examination in the diagnosis of schwannomatosis has been well described in the extant literature[4]. However, reports cover both imaging-ultrasound (US), computed tomography (CT) and magnetic resonance (MR)-and pathological characteristics of schwannomatosis. In this study, we reviewed 12 cases of schwannomatosis seen over the past two decades at our institution, analyzing their clinical, imaging and pathological 
characteristics, and attempted to elucidate the internal relationship between imaging and pathology in schwannomatosis.

\section{Clinical Data And Methods}

This study was a retrospective analysis of 12 cases of schwannomatosis confirmed by pathology after complete imaging examination (US, CT and MRI) at our hospital during January 2000-December 2019. The median age at diagnosis was 39.4 years (range, 22-56 years), with a median delay from the initial symptoms to diagnosis of 7.4 years (range, $0.1-40$ years). All 12 patients received imaging examinations. Two cases were intracranial, 7 involved the intraspinal nerves, 6 were in the torso, 11 were in the limbs, 5 were in the chest and 7 were abdominal. The total number of masses was $>60$ (some masses had fused with one another, making them difficult to accurately count). The focus of imaging observation and pathological diagnosis was the masses that underwent surgical resection or puncture biopsy. (Intracranial and intraspinal masses are not easily examined by US and were therefore not included in the selective analysis). Ultimately, we included a total of 36 masses in our analysis (Table 1).

\section{Table 1 General clinical data of 12 patients with schwannomatosis}

\begin{tabular}{|llllllll|}
\hline & $\begin{array}{l}\text { Male/ } \\
\text { Female } \\
(\mathrm{n})\end{array}$ & $\begin{array}{l}\text { Median } \\
\text { age }(\mathrm{y})\end{array}$ & $\begin{array}{l}\text { Course } \\
\text { of } \\
\text { disease } \\
\text { (y) }\end{array}$ & $\begin{array}{l}\text { Observations/ } \\
\text { Total }\end{array}$ & $\begin{array}{l}\text { Symptomatic/ } \\
\text { Asymptomatic }\end{array}$ & $\begin{array}{l}\text { Resectioned } \\
\text { cases } \\
\text { (masses) }\end{array}$ & $\begin{array}{l}\text { Puncture } \\
\text { cases } \\
\text { (masses) }\end{array}$ \\
\hline Statistic & $10 / 2$ & 39.4 & 7.4 & $36 / 60+$ & $9 / 3$ & $10(32)$ & $2(4)$ \\
\hline
\end{tabular}

\subsection{Inclusion criteria}

(1) The presence of $\geq 2$ non-intradermal schwannomas in different parts of the body as confirmed by pathology (surgery/puncture biopsy), (2) the patient did not meet any existing diagnostic criteria for NF2 or have any first-degree relatives with NF2, (3) blood samples of patients age $<20$ years were genetically tested, and NF2 mutation was found to be absent from blood deoxyribonucleic acid (DNA), and (4) patients age $\leq$ 40 years underwent enhanced MRI, excluding those with bilateral vestibular schwannoma[5, 6].

\subsection{Imaging and pathological-examination methods}

\subsubsection{Ultrasonic-examination method}

We used a GE LOGIQ E9 US system (GE Healthcare, Chicago, IL, USA) and a Canon Aplio i800 color Doppler US diagnostic instrument (Canon Medical Systems USA, Tustin, CA, USA). The probe frequency was 5-12 $\mathrm{MHz}$; for the abdominal convex array probe, the frequency was 2-5 MHz. Two deputy chief US specialists observed schwannomatosis patients' US imaging data. We recorded tumor location, quantity, size, shape, boundary, internal and rear echo, relationship with peripheral nerves and blood vessels, and blood flow signals. When opinions differed, a consensus was reached after discussion. Evaluation criteria were as follows: Blood flow signal in the mass was graded on the Adler scale: grade $\mathbf{0}$, no blood flow signal found in the tumor; grade I, small amount of blood flow, with one to two punctate or rod-shaped tumor blood vessels 
visible; grade II, medium amount of blood flow, with three to four punctate blood vessels visible; and grade III, large amount of blood flow, with at least five punctate blood vessels or two longer blood vessels.

\subsubsection{CT examination method}

We used a Siemens SOMATOM Emotion multi-row spiral CT scanner (Siemens AG, Munich, Germany) with layer thickness of 5-10 mm, layer spacing of $5 \mathrm{~mm}$, window width of 200-350 HU, window position of 35$50 \mathrm{HU}, 1.25-\mathrm{mm}$ thin-layer reconstruction of the focus layer, $120 \mathrm{kV}$ and $220 \mathrm{mAs}$. Meglumine dimeglumine $(300 \mathrm{mg} / \mathrm{ml})$ was used as the contrast agent; we injected it into the elbow vein using a high-pressure syringe at a dosage of $80-120 \mathrm{ml}$ or $1.5 \mathrm{ml} / \mathrm{kg}$ body weight and an injection rate of $2.0-4.0 \mathrm{ml} / \mathrm{s}$. Times of twophase contrast-enhanced scanning were 25-30 s for the arterial phase and $1 \mathrm{~min}$ for the venous phase after injection of contrast medium. Two senior radiologists analyzed the CT manifestations of the lesions, including location, size, shape, number, growth mode, edge, scan density and enhancement characteristics.

\subsubsection{MRI examination method}

We used a GE Healthcare Signa 1.5T superconducting MR machine to scan sequences in the transverse, coronal and sagittal planes. Scanning sequences and parameters were as follows: T1-weighted image (T1WI) repetition time/echo time (TR/TE), 400-500/15-30 ms; T2WI TR/ TE, 500-3800/100-120 ms; T2WI-short-tau inversion recovery (STIR) TR/TE, 5875/80 ms; field of view (FOV), $50 \mathrm{~cm}^{2}$; echo train length, 25; matrix, $320 \times 240$; layer thickness, 5-10 mm; and spacing, $0.5 \mathrm{~mm}$. The contrast agent was gadoliniumdiethylenetriaminepentacetate (Gd-DTPA), the dose was $0.1 \mathrm{mmol} / \mathrm{kg}$ and the injection rate was $2.0 \mathrm{ml} / \mathrm{s}$. Subsequently, we performed an enhanced T1WI scan.

\subsubsection{Pathological-examination method}

All of the specimens were fixed with $4 \%$ neutral formaldehyde, embedded in paraffin and stained with hematoxylin and eosin (H\&E) for histological observation. Section thickness was $4 \mu \mathrm{m}$. We observed the histological morphology of the tumor under an Olympus BX53 light microscope (Olympus, Tokyo, Japan). We used the SP method for immunohistochemical (IHC) staining and the heat repair method for antigen repair. The first antibodies used included S-100, vimentin, calretinin, epithelial-membrane antigen (EMA), Clusters of Differentiation 34 and 56 (CD34, CD56), Ki-67 labeling index (LI), dendritic-cell sarcoma (DCS), smooth-muscle actin (SMA) and mindbomb E3 ubiquitin protein ligase 1 (MIB1).

\subsection{Statistical Methods}

We used SPSS statistical software version 20.0 (IBM Corp., Armonk, New York, USA) for analysis. Considering the small sample size, data for patient age and course of disease were represented by the median. We used the Kolmogorov-Smirnov method to test the normal distribution of quantitative data (tumor diameter). Measurement data conforming to a normal distribution were expressed by.

\subsection{Ethics Statement}


This retrospective study was approved by our hospital's institutional review board. We obtained signed consent from each patient.

\section{Results}

\subsection{Ultrasonic-sonogram features}

According to their US characteristics, the 36 masses could be roughly divided into 3 categories: (1) Solid, with a uniform elliptical or circular shape and a clear edge. Nineteen masses were solid, of which 16 had an envelope and 3 did not. Eleven of the 19 had uneven low echo; The other 8 masses had a uniform low echo. (2) Cystic. Five masses fell into this category. The wall of the cyst was smooth and most of the non-echoic areas were in the cyst. A small number of point and floccule hyperechoic areas were seen, some accompanied with strip hyperechoic separation, and the echo at the rear was obviously enhanced. (3) Cystic-solid (mixed) type, of which there were 12 masses. Tumor volume was generally large, the mass was round or oval and its membrane was intact. There were gridlike or irregular flake-like anechoic areas in the interior, and the echo in the rear was slightly enhanced. US features of the 36 masses are shown in Table 2.

\subsection{CT imaging features}

Internal mass density on CT plain scan was uniform in 9 masses and uneven in 27. On CT plain scan, density was mainly medium and low, slightly lower than that of muscle. CT value was 15-30 HU. After intravenous injection of contrast agent, the enhanced scan was mainly light and medium inhomogeneous, and enhanced CT value was 20-45 higher than on plain scan. We found heterogeneous reticular enhancement, clear boundary.

\subsection{MR image features}

Of the 36 masses, 12 were round (33.33\%), 21 were oval (58.33\%) and 3 were dumbbell-shaped (8.33\%). Mass size ranged from 1.5 to $14 \mathrm{~cm}$ (average, $5.2 \pm 0.8 \mathrm{~cm}$ ). Masses were mainly low and equal signal on T1WI but were dominated by an uneven high signal on T2WI. Tumors showed obvious uniform or uneven enhancement. Most of the capsule was present, and enhancement of the tumor capsule was not obvious on MRI enhanced scan. The long axis of the tumor ran along the direction of the nerve, from the proximal to the distal end; the surrounding tissues were compressed and changed, and the destruction was not obvious.

\subsection{Pathological features}

Most of the masses were round or nodular, tough and with complete capsules. Cystic change and necrosis were seen in 17 masses. Under the light microscope, we saw 2 kinds of tumor tissue component manifestation: the fusiform-sheath tumor cells were in a paliform or interlaced arrangement, and some cells in the Antoni A area, loose distribution of cells in the Antoni B area, vascular proliferation and cyst formation. According to the proportions of both Antoni areas, the 36 masses could be divided into 4 types: type I:

Central Antoni A area, peripheral Antoni B area, 10 masses (27.8\%); type II: mainly Antoni A area with uniform distribution, 9 masses (25.0\%); type III: mainly Antoni B area, 5 masses (13.9\%); and type IV: hybrid of Antoni 
A and $B$ areas, 12 masses (33.3\%). Positive expression of CD34 was $46.7 \%$ with S-100 $(+++)$. The Ki-67 proliferation index was low $(1-5 \%)$.

2.5 Relationship between imaging and histopathology features see Table 3 and Figures 1-4 for a typical case (40 years old patient, male)

Table 2 Ultrasonic-sonogram features of 36 masses

\begin{tabular}{|c|c|c|c|c|c|c|c|}
\hline Features & $\begin{array}{l}\text { Shape } \\
\text { (n) }\end{array}$ & $\begin{array}{l}\text { Boundary } \\
\text { (n) }\end{array}$ & $\begin{array}{l}\text { Type } \\
\text { (n) }\end{array}$ & $\begin{array}{l}\text { Target } \\
\text { sign } \\
(n)\end{array}$ & $\begin{array}{l}\text { Tail } \\
\text { sign } \\
\text { (n) }\end{array}$ & $\begin{array}{l}\text { Neurovascular } \\
\text { concomitant sign } \\
\text { (n) }\end{array}$ & $\begin{array}{l}\text { CDFI Adler } \\
\text { classification } \\
\text { (n) }\end{array}$ \\
\hline \multirow[t]{4}{*}{ Statistic } & $\begin{array}{l}\text { Circular } \\
(12)\end{array}$ & $\begin{array}{l}\text { Clear } \\
(33)\end{array}$ & $\begin{array}{l}\text { Solid } \\
\text { (19) }\end{array}$ & Y (19) & $\begin{array}{l}Y \\
(20)\end{array}$ & $Y(14)$ & Grade 0 (3) \\
\hline & $\begin{array}{l}\text { Ellipse } \\
(21)\end{array}$ & $\begin{array}{l}\text { Unclear } \\
\text { (3) }\end{array}$ & $\begin{array}{l}\text { Cystic- } \\
\text { solid } \\
(12)\end{array}$ & $\mathrm{N}(17)$ & $\begin{array}{l}N \\
(16)\end{array}$ & $N(22)$ & Grade I (9) \\
\hline & $\begin{array}{l}\text { Dumbbell } \\
\text { (3) }\end{array}$ & & $\begin{array}{l}\text { Cystic } \\
\text { (5) }\end{array}$ & & & & Grade II (17) \\
\hline & & & & & & & Grade III (7) \\
\hline
\end{tabular}

Abbreviations: $\mathrm{Y}=\mathrm{YES} ; \mathrm{N}=\mathrm{NO}$

Table 3 Relationship between imaging features and histopathology of 36 masses 


\begin{tabular}{|c|c|c|c|c|}
\hline $\begin{array}{l}\text { Pathological } \\
\text { type }\end{array}$ & Type I (n) & Type II (n) & Type III (n) & Type IV (n) \\
\hline $\begin{array}{l}\text { Number of } \\
\text { masses }\end{array}$ & 10 & 9 & 5 & 12 \\
\hline \multirow[t]{5}{*}{ US features } & Solid & Solid & Cystic & Cystic-solid \\
\hline & $\begin{array}{l}\text { Inhomogeneous echo } \\
\text { target sign (7) }\end{array}$ & $\begin{array}{l}\text { Homogeneous } \\
\text { echo }\end{array}$ & $\begin{array}{l}\text { Honeycombed liquid } \\
\text { dark area }\end{array}$ & $\begin{array}{l}\text { Inhomogeneous } \\
\text { low echo }\end{array}$ \\
\hline & CDFI grade: & CDFI grade: & CDFI grade: & CDFI grade: \\
\hline & \multirow{2}{*}{ II (6) } & I (3) & $0(3)$ & II (5) \\
\hline & & II (6) & I (2) & III (7) \\
\hline \multirow[t]{6}{*}{ CT features } & $\begin{array}{l}\text { Pre-contrast: } \\
\text { Inhomogeneous }\end{array}$ & $\begin{array}{l}\text { Pre-contrast: } \\
\text { Homogeneous }\end{array}$ & $\begin{array}{l}\text { Pre-contrast: } \\
\text { Inhomogeneous (high } \\
\text { external/low internal) }\end{array}$ & $\begin{array}{l}\text { Pre-contrast: } \\
\text { Inhomogeneous }\end{array}$ \\
\hline & $\begin{array}{l}\text { Post-contrast } \\
\text { enhancement: }\end{array}$ & $\begin{array}{l}\text { Post-contrast } \\
\text { enhancement: }\end{array}$ & $\begin{array}{l}\text { Post-contrast } \\
\text { enhancement: }\end{array}$ & $\begin{array}{l}\text { Post-contrast } \\
\text { enhancement: }\end{array}$ \\
\hline & Internal mild (4) & Mild (4) & & \multirow{2}{*}{$\begin{array}{l}\text { Solid area: } \\
\text { Uneven (12) }\end{array}$} \\
\hline & Internal moderate (4) & Moderate (3) & 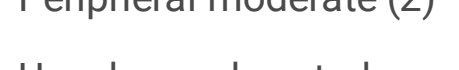 & \\
\hline & \multirow[t]{2}{*}{ Internal severe (2) } & \multirow[t]{2}{*}{ Severe (2) } & $\begin{array}{l}\text { Unenhanced central } \\
\text { low-density area (2) }\end{array}$ & $\begin{array}{l}\text { (CT value } \\
\text { increased by } 20- \\
45 \mathrm{HU} \text { ) }\end{array}$ \\
\hline & & & & $\begin{array}{l}\text { Cystic area: } \\
\text { Unenhanced (12) }\end{array}$ \\
\hline \multirow[t]{4}{*}{$\begin{array}{l}\text { MRI } \\
\text { features }\end{array}$} & \multirow{4}{*}{$\begin{array}{l}\text { T1: Low signal } \\
\text { T2: High signal } \\
\text { Enhanced T1: Center } \\
\text { enhancement, edge } \\
\text { not enhanced }\end{array}$} & \multirow[t]{2}{*}{$\begin{array}{l}\text { T1: Low, equal } \\
\text { signal (hybrid) }\end{array}$} & T1: Much lower signal & \multirow[t]{2}{*}{$\begin{array}{l}\text { T1: Low, hybrid } \\
\text { signal }\end{array}$} \\
\hline & & & T2: Much higher signal & \\
\hline & & $\begin{array}{l}\text { T2: High, } \\
\text { equal signal } \\
\text { (hybrid) }\end{array}$ & \multirow[t]{2}{*}{$\begin{array}{l}\text { Enhanced T1: Obvious } \\
\text { uneven enhancement }\end{array}$} & $\begin{array}{l}\text { T2: Slightly high, } \\
\text { hybrid signal }\end{array}$ \\
\hline & & $\begin{array}{l}\text { Enhanced T1: } \\
\text { Uniform } \\
\text { enhancement }\end{array}$ & & $\begin{array}{l}\text { Enhanced T1: } \\
\text { Obvious uneven } \\
\text { enhancement }\end{array}$ \\
\hline
\end{tabular}

Note: Type I: Central Antoni A area, peripheral Antoni B area; Type II: Mainly Antoni A area with uniform distribution; Type III: Mainly Antoni B area; Type IV: Hybrid of Antoni A and B areas.

\section{Discussion}

3.1 Schwannomatosis is a benign tumor that is characterized by multiple peripheral schwannomas with central nervous-system (CNS) tumors and various defects of the nervous system. About $30 \%-40 \%$ of patients carry LZTR1 mutations[7]. The tumorigenesis of schwannomatosis is a response to a somatic fivehit/three-step mechanism, resulting in the loss of function of genes adjacent to LZTR1 and the contiguous 
genes of locus 22q11.2q12.2[8]. Paganini et al.[9] immunostained for LZTR1 protein in 22 masses from nine unrelated patients. It is suggested that LZTR1 molecular analysis might help elucidate the molecular characteristics of schwannomatosis patients. Ding et al.[10] suggest that the structural and functional abnormalities of the SMARCB1 gene might be the molecular basis of familial schwannomatosis. If the focus involves peripheral-nerve tissue, it can be accompanied by pain[11]. Jordan et al.[12], using a simple 10-point pain scale (SF-36) to explore the relationship between pain and mutation of the SMARCB1 and LZTR1 genes in schwannomatosis, found that the median pain score of the LZTR1 group was 3.9 and that of the $S M A R C B 1$ group was $0.5(P=0.0414)$. Not only was the pain of $L Z T R 1$ mutation patients significantly higher than that of SMARCB1 mutation patients, but their pain-related quality of life as assessed by SF-36 was significantly worse $(P=0.0106)$. Pain score was correlated with tumor volume (rho $=0.32471, P=0.0499)$ but not with number of tumors (rho $=0.23065, P=0.1696$ ). Nervous-system defects include hypoacusis, tinnitus and amyotrophy. When the CNS tumor exerts pressure on the surrounding tissues, symptoms of sensory and motor disorders can appear. The course of disease is chronic and benign. Histopathological features are as follows: the tumor tissue has an intact capsule; the tumor body is a single, spindle-shaped Schwann cell; and the tumor cell mass is separated by the stroma and has thick blood vessels around it. In clinical practice, doctors should consider a diagnosis of schwannomatosis in patients with multiple schwannomas and perform an active physical examination and imaging examination of the nervous system. It is worth noting that Mehta et al.[13] believe that schwannomatosis patients without LZTR1 mutation can have unilateral vestibular schwannoma. Asai et al.[14] also reported a case of pathologically confirmed left-cerebellopontine vagal-schwannoma tumor in whose family members a diagnosis of NF1 or NF2 was ruled out; genetic analysis revealed a germline mutation of SMARCB1. Smith et al.[15] also believe that patients with schwannomatosis may have vestibular schwannoma, which should not be considered an exclusion criterion for clinical diagnosis. However, Baruah et al.[16] suggest that schwannomatosis should be excluded if MRI shows any vestibular schwannoma. Radek et al.[17] also believe that the clinical features of schwannomatosis are similar to NF2 but that there are no vestibular schwannomas in schwannomatosis. The previous diagnostic standard ruled out schwannomatosis if there were a history of the bilateral vestibular neuroma, but whether unilateral vestibular neuroma can exclude schwannomatosis is worth further exploration.

One of the main obstacles in the study of schwannomatosis is the lack of robust tumor cell lines. At present, there is no suitable tool for studying the mechanism of and conducting drug discovery for schwannomatosis. The current standard treatment is still surgical resection[18].

\subsection{Imaging features of schwannomatosis}

\subsubsection{Ultrasonic characteristics}

In this study, US examination often showed masses distributed along the nerve. Most were oval (21/36) or round (12/36) low-echo masses with enhanced shadows in the posterior of the mass. Fused tumors could be distributed in a beadlike fashion. Because of the different pathological components of masses, the internal echo could be homogeneous (9/36) or inhomogeneous (27/36). Borders were mostly clear, and shapes were regular. CDFI showed mainly point and strip blood flow signals, with Adler grade II (15/36) the most 
common. The opposite ends of each mass were connected by nerve fibers, and a tail sign (20/36) was found in some masses.

\subsubsection{CT characteristics}

Masses were located in the muscle space; there was a slightly low-density, oval-shaped block shadow running longitudinally; and density could be equal or unequal. Enhancement scanning varied according to the pathological components of the tumor. In type II tumors, CT enhancement masses were almost uniformly enhanced; there was a mild enhancement in four cases, moderate and severe enhancement in 1 case each. The CT enhancement of type I, III and IV tumors was mainly unequal. Type I showed mainly central enhancement. Type III showed mainly peripheral light-to-moderate enhancement, and two of these five masses had no enhancement due to the cystic area in the center. All of the type IV tumors showed inhomogeneous enhancement, and their CT values increased by $20-45 \mathrm{HU}$.

\subsubsection{MRI characteristics}

The main MRI manifestations of masses in schwannomatosis were low and equal signal on T1W1, medium and high signal on T2W1 and mixed high signal on diffusion-weighted imaging (DWI). Edge and internal continuous enhancement can be seen on the enhanced scan, and the boundary between benign tumors and surrounding tissue is relatively clear[19]. The results of this study were similar to those described in the extant literature. MRI is the most effective imaging diagnostic method for intraspinal tumors, as it can clearly distinguish various tissues and structures in the spinal canal and it has certain use for determining the origin, shape, size, quantity and adjacent structures of tumors. It can also be used to guide the formulation of the surgical plan. The whole spine must be scanned via MRI before intraspinal-tumor surgery; doctors must carefully observe the small foci to avoid a missed diagnosis.

\subsubsection{Advantages and disadvantages of different imaging modalities}

Because US examination is inexpensive, very safe, convenient, easy to operate and without risk of radiation damage, it has become the first choice for screening and postoperative reexamination of schwannomatosis. However, because of the bone block, US examination is limited in the detection of intracranial and intraspinal lesions. CT has high spatial resolution and no overlapping of tissue structure images, allowing it to accurately judge the relationship between masses and surrounding tissues, which provides an important basis for clinical surgery; however, its soft-tissue resolution is poor. MRI has the advantages of high softtissue resolution and multi-directional imaging, which can better show the source of the tumor, as well as the relationship between masses and blood vessels/surrounding tissues; it is more conducive to differentiating between benign and malignant tumors, so it has become a prerequisite examination before surgery. Godel et al.[20] compared the volumes of dorsal-root ganglia in 16 patients with schwannomatosis, 14 patients with NF2 and 26 healthy controls by MR neurography. Their study found that dorsal-root ganglion volume was the same in NF2 as in healthy controls, but not in schwannomatosis. Dorsal-root ganglia might be vulnerable sites in terms of origination of areflexia and sensory loss, as well as useful diagnostic markers in NF2. MRI examination is costlier than ultrasound and $\mathrm{CT}$, and it is not suitable for patients who have nondemagnetized metal implants or claustrophobia. In addition, in recent years, some researchers have used 
positron emission tomography (PET) for imaging diagnosis of schwannomatosis[21], but PET imaging might not be a reliable predictor of malignant transformation in schwannoma, which reduces the enthusiasm for tumor surgery without obvious clinical symptoms or signs[22]. This imaging modality is also expensive and difficult to promote.

\subsubsection{Pathological features of schwannomatosis}

In general, specimens have a capsule, and there are no nerve fibers in the tumor body. In terms of path morphology, two types of schwannoma tissue can be seen under the microscope. In type A tissue, tumor cells are arranged in bundles; cells within the tissue are spindle-shaped and arranged parallel to each other into palisades; the nucleus is thin and long; and there are abundant slender reticular fibers between the cells. Type $B$ has few cells in the tissue. The arrangement of tumor cells is disordered and loose, with liquid between cells and fibers that can collect in the capsule. There is a large extracellular space between tissues, and abundant capillaries and blood sinuses between cells. Tumor blood vessels often show sinusoidal, spongy, or capillary-like dilation and hyaline change of blood vessel walls. In terms of immunophenotype, schwannomatosis is the same as classic soft-tissue schwannoma. Our 12 cases all showed diffuse positive S-100 protein expression, which could be used as the first marker. NF was generally not expressed, Ki-67 was below $3 \%$, and patients were mostly positive for CD34 and vimentin.

\subsubsection{Relationship between imaging manifestations and pathology}

This study showed a certain correlation between tumor imaging and pathological properties in schwannomatosis. Because of the density of the cells in Antoni type A tissue, CT plain scan and US findings in lesions mainly composed of this tissue showed mostly moderate and slightly low density or echo. The uniformity of the internal density or echo depended on the amount of Antoni type B tissue. The distribution of Antoni type B tissue in the tumor was consistent with that of the low-density and low-echo areas. Antoni B areas easily become cystic, and their imaging features are round low-density areas with smooth inner walls. The enhancement range of the tumor varied greatly, from light to significant; the enhancement could be irregular or more uniform, or there could be interstitial or cystic low density in the enhancement area. This change was consistent with the distribution of multicystic space and hemorrhagic degeneration in Antoni type B tissue, similar to the characteristic manifestation of schwannoma on T2Wl: target sign. In this study, 25 masses had enhancement amplitudes $>20 \mathrm{HU}, 5$ of which were schwannomas with active growth. There were abundant blood sinuses in the tumors. The blood-rich Antoni type A tissues were enhanced, while enhancement of Antoni type B tissues was not obvious. In this study, 11 masses with enhancement $<20 \mathrm{HU}$ were found to have a large number of collagen fibers. Other causes of tumor enhancement included hyaline degeneration, hemorrhage and necrosis. CDFI revealed no significant blood flow signal in most lesions, only a small amount of linear color blood flow signal. The reason was that the wall of the tumor vessel was fibrosis and accompanied by thrombosis.

\subsection{Differential diagnosis}

Neurofibromatosis type 20NF2 
$\mathrm{NF} 2$ is an autosomal-dominant inherited disease that is characterized by multiple benign tumors of the nervous system and caused by a gene located on the q12 band of chromosome 22. Bilateral acoustic neuroma, the first symptom of which is bilateral progressive hearing loss, is the most common clinical characteristic of NF2. Clinical diagnosis of NF2 is based on the Manchester standard[23]. There is a certain overlap between the diagnoses of schwannomatosis and NF2, the characteristics of the 2 conditions differ nonetheless: (1) they have different prevalence rates. Evans et al.'s[1] epidemiological survey found that the prevalence of schwannomatosis is one in 126,315; that of NF2, 1 in 50,500. The calculated birth incidences were one in 68,956 and one in 27,956 , respectively. (2) The onset age of NF2 is generally $<30$ years, while schwannomatosis is more common in the age range of 20-50 years. (3) NF2 often involves bilateral vestibular nerves, while schwannomatosis involves the skin with multiple plexiform nerves. NF2 patients also tend to develop meningiomas and spinal ependymomas, while schwannomatosis patients rarely develop intracranial meningiomas or bilateral vestibular schwannoma[15]. (4) Life expectancy in schwannomatosis (mean age at death, 76.9 years) is significantly better than in NF2 (mean age at death, 66.2 years)[1]. (5) Farschtschi et al.[24] measured the intraepidermal nerve fiber density (IEND) of skin biopsies and found that the IEND of patients with schwannomatosis (97\%) was significantly lower than the standard reference, while fewer than half (44\%) of NF2 patients had such low IEND. (6) Louvrier et al.[25] suggested that the key to differential diagnosis between NF2 and schwannomatosis is the use of an amplification-based method to sequence tumor suppressor genes in NF2: SMARCB1, LZTR1, SWI/SNF-related matrix-associated actindependent regulator of chromatin subfamily E member 1 (SMARCE1) and suppressor of fused homolog (SUFU). (7) Schwannomatosis is mainly composed of Schwann cells, while the cells that comprise NF2 include Schwann cells, nerve bundle membrane-like cells, fibroblasts and intermediate cells. (8) Nerve fibers often pass through NF2 masses but not through those seen in schwannomatosis. (9) Schwannomatosisassociated schwannomas tend to exhibit greater peripheral edema, and the myxoid change in the focus shows T2 high signal. Moreover, the range of minimum apparent diffusion coefficient (ADC) values is $0.8-$ 2.7 in NF2, 0.3-2.2 in schwannomatosis[4].

\subsection{Treatment and prognosis}

Surgical resection is the most reliable treatment for schwannomatosis. Ten of our 12 patients underwent surgery. The other two declined the operation after communication with us because they had too many tumors and surgery would have been difficult.

\subsection{Study limitations}

The limitations of this study were as follows: (1) This was a retrospective study with a small number of cases. Because some of the diagnoses were confirmed at an early age and/or before the new diagnostic standard came into being[26], not all of the patients underwent Iztr1 and smarcb1 gene examination for clinical diagnosis. We cannot guarantee that any of the patients with schwannomatosis did not also have mosaic NF2. Some patients with mosaic NF2 will be included in this diagnosis when they are at a young age. Some patients with schwannomatosis might have unilateral vestibular schwannoma. (2) These patients did not have 3-mm thin slice scans or whole-body MRI examinations, and it is not excluded that some masses were not found in some areas without scanning. (3) It was difficult for some patients to confirm their family histories or for us to obtain samples from their family members for genetic analysis. (4) We did not compare 
the imaging characteristics seen in this study with those of NF2. (5) Because treatment plan and efficacy evaluation were not the focus of this study, no corresponding discussion and case follow-up analysis was conducted.

\section{Declarations}

Data Availability Statement Some or all data, models, or code generated or used during the study are available from the corresponding author by request. (liuinsistence@163.com).

Author contributions Cheng-wei Kang $₫$ Responsible for research design, literature review, statistical analysis, and article writing $\mathbb{}$ Chang-chao Dong, Gang Tan, Zhao-kui Yan, Xiao-ping Pu囚Collect and sort out relevant

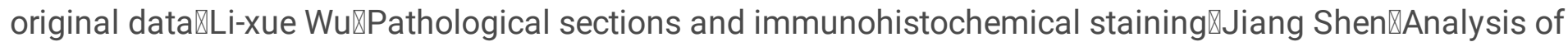
imaging characteristics $\llbracket$ Lei LiuखResponsible for the revision of articles.

Funding Not applicable.

\section{Compliance with ethical standards}

Conflict of interest We declare that we have no financial and personal relationships with other people or organizations that can inappropriately influence our work, there is no professional or other personal interest of any nature or kind in any product, service, and/or company that could be construed as influencing the position presented in this article.

\section{References}

1. Evans DG, Bowers NL, Tobi S et al (2018) Schwannomatosis: a genetic and epidemiological study. J Neurol Neurosurg Psychiatry 89(11):1215-1219

2. Antinheimo J, Sankila R, Carpén O, Pukkala E, Sainio M, Jääskeläinen J (2000) Population-based analysis of sporadic and type 2 neurofibromatosis-associated meningiomas and schwannomas. Neurology 54(1):71-76

3. Merker VL, Esparza S, Smith MJ, Stemmer-Rachamimov A, Plotkin SR (2012) Clinical features of schwannomatosis: a retrospective analysis of 87 patients. Oncologist 17(10):1317-1322

4. Ahlawat S, Blakeley JO, Langmead S, Belzberg AJ, Fayad LM (2020) Current status and recommendations for imaging in neurofibromatosis type 1 , neurofibromatosis type 2 , and schwannomatosis. Skeletal Radiol 49(2):199-219

5. Baser ME, Evans DG, Friedman JM (2006) Increasing the specificity of diagnostic criteria for schwannomatosis. Neurology: Official Journal of the American Academy of Neurology. (5): 730-732

6. Plotkin SR, Blakeley JO, Evans DG et al (2013) Update from the 2011 International Schwannomatosis Workshop: From genetics to diagnostic criteria. Am J Med Genet A 161A(3):405-416

7. Kehrer-Sawatzki H, Farschtschi S, Mautner VF, Cooper DN (2017) The molecular pathogenesis of schwannomatosis, a paradigm for the co-involvement of multiple tumour suppressor genes in tumorigenesis. Hum Genet 136(2):129-148

Page $13 / 18$ 
8. Deiller C, Van-Gils J, Zordan C et al (2019) Coexistence of schwannomatosis and glioblastoma in two families. Eur J Med Genet 62(8):103680

9. Paganini I, Chang VY, Capone GL et al (2015) Expanding the mutational spectrum of LZTR1 in schwannomatosis. Eur J Hum Genet 23(7):963-968

10. Ding Y, Rong H, Wang Y et al (2019) Detection of Germline Mutations of the SMARCB1 Gene in a Chinese Family with Intraspinal Schwannomatosis. World Neurosurg 123:318-322

11. Ostrow KL, Donaldson KJ, Caterina MJ, Belzberg A, Hoke A (2019) The Secretomes of Painful Versus Nonpainful Human Schwannomatosis Tumor Cells Differentially Influence Sensory Neuron Gene Expression and Sensitivity. Sci Rep 9(1):13098

12. Jordan JT, Smith MJ, Walker JA et al (2018) Pain correlates with germline mutation in schwannomatosis. Med (Baltim) 97(5):e9717

13. Mehta GU, Feldman MJ, Wang H, Ding D, Chittiboina P (2016) Unilateral vestibular schwannoma in a patient with schwannomatosis in the absence of LZTR1 mutation. J Neurosurg 125(6):1469-1471

14. Asai K, Tani S, Mineharu Y et al (2015) Familial schwannomatosis with a germline mutation of SMARCB1 in Japan. Brain Tumor Pathol 32(3):216-220

15. Smith MJ, Kulkarni A, Rustad C et al (2012) Vestibular schwannomas occur in schwannomatosis and should not be considered an exclusion criterion for clinical diagnosis. Am J Med Genet A 158A(1):215219

16. Baruah RK, Bora S, Haque R (2016) Segmental Schwannomatosis of the Spine: Report of a Rare Case and Brief Review of Literature. Ortop Traumatol Rehabil 18(1):73-78

17. Radek M, Tomasik B, Wojdyn M, Snopkowska-Wiaderna D, Błaszczyk M, Radek A (2016) Neurofibromatosis type 2 (NF 2) or schwannomatosis?-Case report study and diagnostic criteria. Neurol Neurochir Pol 50(3):219-225

18. Ostrow KL, Donaldson K, Blakeley J, Belzberg A, Hoke A (2015) Immortalized Human Schwann Cell Lines Derived From Tumors of Schwannomatosis Patients. PLoS One 10(12):e0144620

19. Caltabiano R, Magro G, Polizzi A et al (2017) A mosaic pattern of INI1/SMARCB1 protein expression distinguishes Schwannomatosis and NF2-associated peripheral schwannomas from solitary peripheral schwannomas and NF2-associated vestibular schwannomas. Childs Nerv Syst 33(6):933-940

20. Godel T, Mautner VF, Farschtschi S et al (2018) Dorsal root ganglia volume differentiates schwannomatosis and neurofibromatosis 2. Ann Neurol 83(4):854-857

21. Morshed RA, Lee AT, Lee YM, Chin CT, Jacques L (2019) Schwannomatosis of the Spinal Accessory Nerve: A Case Report. J Brachial Plex Peripher Nerve Inj 14(1):e9-e13

22. Lieber B, Han B, Allen J et al (2016) Utility of positron emission tomography in schwannomatosis. J Clin Neurosci 30:138-140

23. MacCollin M, Chiocca EA, Evans DG et al (2005) Diagnostic criteria for schwannomatosis. Neurology 64(11):1838-1845

24. Farschtschi SC, Kluwe L, Schön G et al (2020) Distinctive low epidermal nerve fiber density in schwannomatosis patients provides a major parameter for diagnosis and differential diagnosis. Brain Pathol 30(2):386-391 
25. Louvrier C, Pasmant E, Briand-Suleau A et al (2018) Targeted next-generation sequencing for differential diagnosis of neurofibromatosis type 2, schwannomatosis, and meningiomatosis. Neuro Oncol 20(7):917-929

26. Kehrer-Sawatzki H, Farschtschi S, Mautner VF, Cooper DN. The molecular pathogenesis of schwannomatosis, a paradigm for the co-involvement of multiple tumour suppressor genes in tumorigenesis. Hum Genet. 136(2): 129-148

\section{Figures}

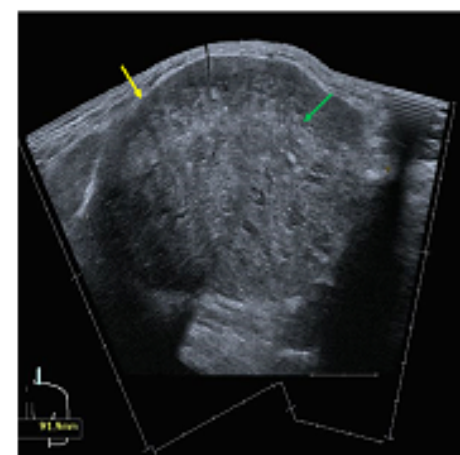

A

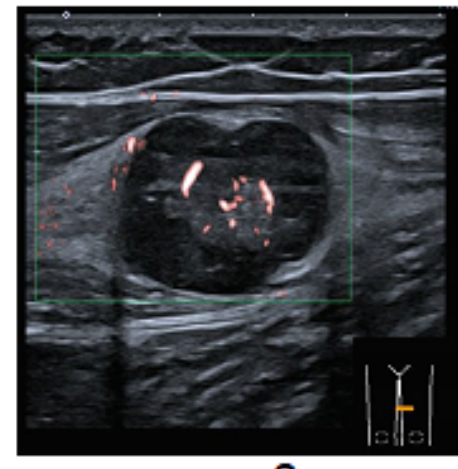

C

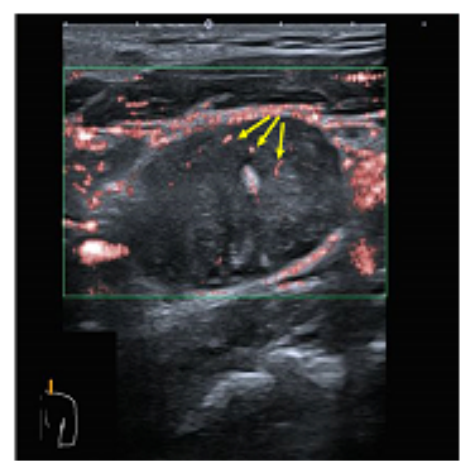

B

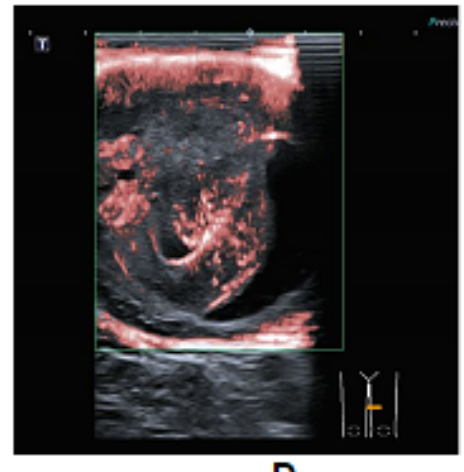

D

\section{Figure 1}

A Left neck mass. Yellow arrow: capsule is complete; Green arrow: Inhomogeneous internal echo. B Left neck mass. Yellow arrow: blood vessels in the mass. CDFI: Small amount of blood flow signal. C Left thigh mass. CDFI: medium flow signal. D Left thigh mass. CDFI: multiple blood flow signals. 


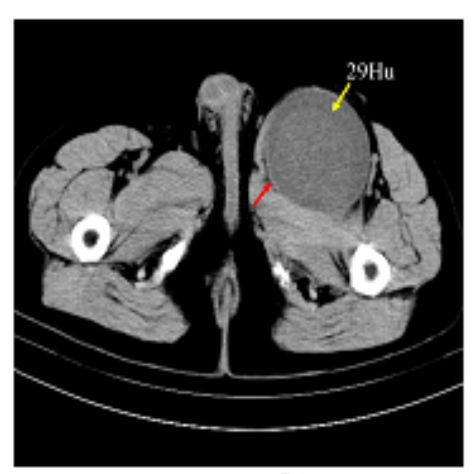

A

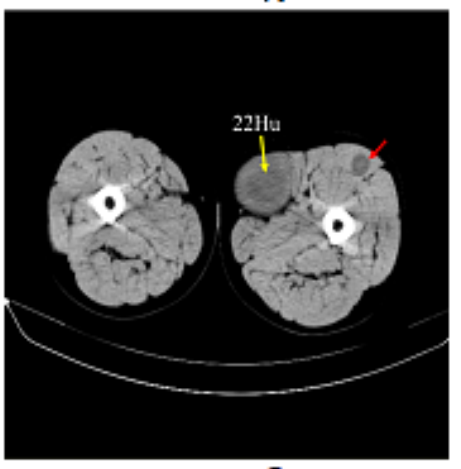

C

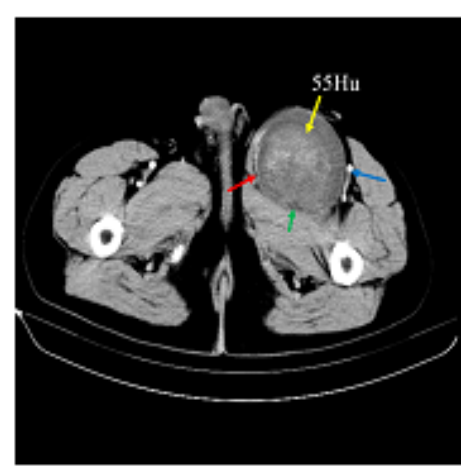

B

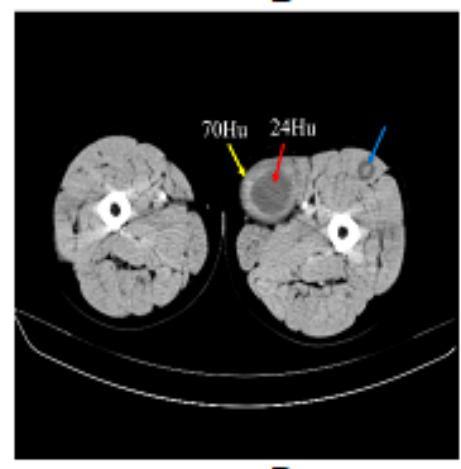

D

\section{Figure 2}

A Left-thigh mass. Plain CT value: 29 HU. Red arrow: clear and complete capsule. Yellow arrow: CT value of the mass on the plain scan. B Left-thigh mass. Arterial-phase $\mathrm{CT}$ value: $55 \mathrm{HU}$. Blue arrow: companion vessel; green arrow: small amount of cystic area. C Mass in left midthigh. Plain CT value: 22 HU. D Mass in left midthigh. Yellow arrow: ring enhancement of the mass. Enhancement CT value of edge: $70 \mathrm{HU}$. Internal enhancement not obvious. Blue arrow: central enhancement of the mass. 

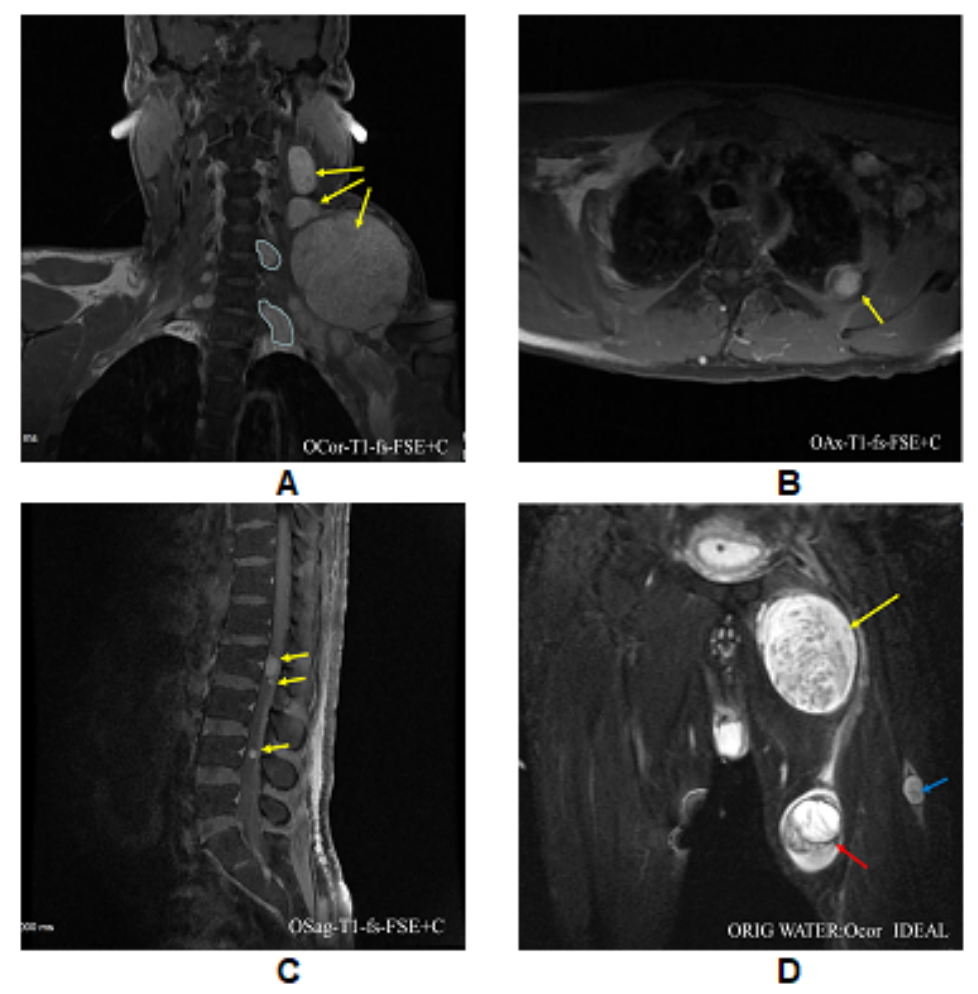

\section{Figure 3}

A Left neck mass. Yellow arrows: clear boundary, distributed along the brachial plexus. Blue-circled areas: the mass is connected with the spinal canal. B Pleural mass along the intercostal nerve, as shown by the yellow arrow. C Lumbar spinal-canal mass. Yellow arrows: along the cauda equina nerve. D ORIG WATER: OCor IDEAL MRI sequence to observe the masses in the left thigh.

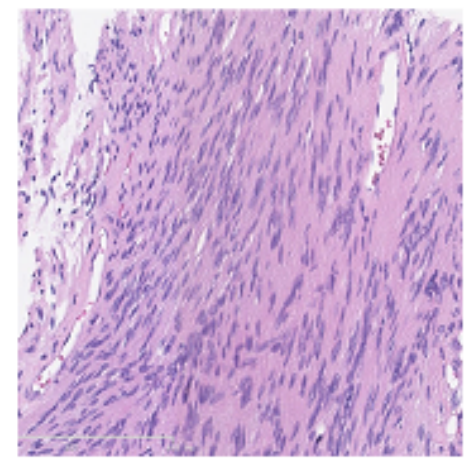

A

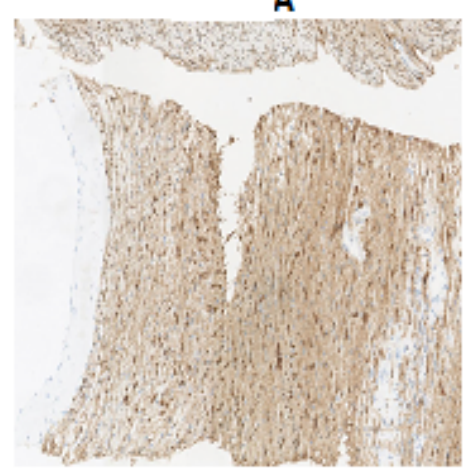

C

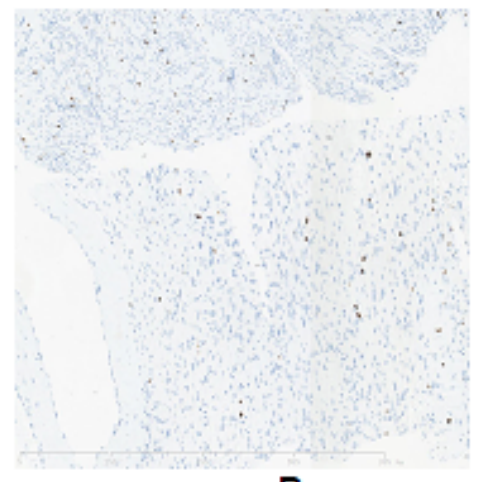

B

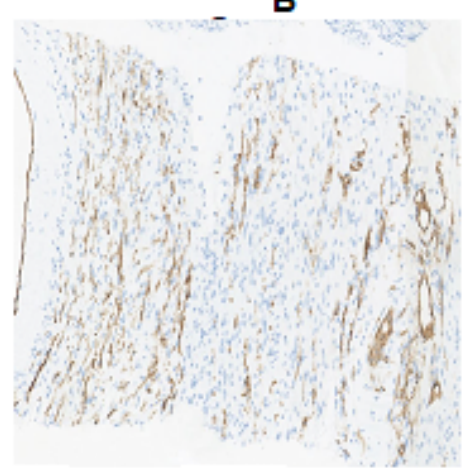

D 


\section{Figure 4}

Left neck mass A HE-200x Mainly Antoni A area with peripheral Antoni B area B MIB1-100x Positive expression of MIB-1 was 5\% C S100-100x Positive expression of S100 D CD34-100x Positive expression of CD34 